\title{
Los "mil rostros" del quechua en el Norte de Potosí ${ }^{1}$
}

Rosaleen Howard

Newcastle University

\begin{abstract}
This article examines the relationship between the Aymara, Quechua and Spanish languages in the central valleys of Bolivia, as this was observed by the author during several fieldtrips to the region in the 1990s. It is based on the premise that the socio-geographic distribution and patterns of use of these languages is best explained in terms of the unequal social, economic and political relations of power that pertained between the urban and rural sectors of society during that period. The article first gives an overview of the sociolinguistic landscape of Northern Potosi. It then proceeds to an analysis of the mutual influences between the three languages, in a series of lexical fields in particular. Using an anthropological linguistic approach, emphasis is placed on speaker perspective and cultural context, in order to explore the significance of words arising from language contact, rather than on their formal features alone.
\end{abstract}

\footnotetext{
1 Este trabajo es una adaptación de Howard-Malverde (1995). Quisiera agradecer la colaboración de los pobladores del pueblo de San Pedro de Buenavista; asimismo, de las comunidades campesinas de Sikuya y Qayarani, durante mis varias estadías en su territorio. También aprecio el apoyo de Mary Jo Dudley, Andrew Canessa y Rodolfo Malverde, que me acompañaron en diferentes visitas. Asimismo, agradezco a Juan de Dios Yapita por la ayuda con el análisis del aimara y a Denise Arnold por su apoyo en cuanto a la terminología del tejido. A Luis Andrade le agradezo por su revisión del texto final. Cualquier error que persista es de mi responsabilidad.
} 


\section{Keywords}

Andean languages, Bolivia, language contact, language ideologies, Northern Potosí

\section{Resumen}

Este artículo examina la interrelación entre las lenguas aimara, quechua y castellano en los valles centrales de Bolivia tal como la autora la observó durante varias estadías de trabajo de campo en los años 1990. Se basa en la premisa de que la distribución socio-geográfica y los patrones de uso de estas lenguas se deben explicar en términos de las relaciones de poder sociales, económicas y políticas desiguales entre el sector urbano y el sector campesino que se daban en la época. En primer lugar, el artículo ofrece una reseña del panorama de la sociolingüística macro, luego procede a un análisis a nivel micro de las influencias entre las tres lenguas, enfocándose en una serie de campos léxicos en particular. Se recalca el interés de tomar en cuenta las perspectivas de los mismos hablantes para calificar el significado del contacto lingüístico desde un punto de vista antropológico y no meramente formal.

\section{Palabras claves}

Bolivia, contacto lingüístico, ideologías lingüísticas, lenguas andinas, Norte de Potosí

\section{Contexto sociolingüístico}

En este artículo quisiera hacer un homenaje al trabajo fundacional que representa el libro Los mil rostros del quechua de Xavier Albó, publicado por el IEP en el Perú en el año 1974, que fue una elaboración de su tesis doctoral de Cornell (Albó 1974). Este libro, como avancé en otro trabajo "enfoca las variables sociales que rigen los patrones de bilingüismo en [Cochabamba] y pone atención a las variaciones dialectales del quechua y sus correlaciones sociales" (Howard 2007, 162). Como estudio a fondo sobre el contacto entre quechua y castellano en un medio urbano, el trabajo de Albó no ha sido superado en el campo de la sociolingüística andina boliviana. Demuestra cómo, en diversos grados, la interpenetración del castellano con el quechua caracteriza el habla de todas las capas sociales en Cochabamba. Los hallazgos 
de Albó también sirven de insumo para los estudios teóricos del bilingüismo y como base para nuevas investigaciones y actualizaciones en el día de hoy. ${ }^{2}$

Inspirada por el trabajo de Albó, voy a tratar la interrelación entre las lenguas castellana, quechua y aimara en la región rural, más precisamente en los valles norcentrales del departamento de Potosí, pertenecientes a la provincia de Charcas, en la zona denominada Norte de Potosí (Fig. 1). Mis datos provienen de varios períodos de trabajo de campo que llevé a cabo en el pueblo de San Pedro de Buenavista (capital cantonal y antiguo pueblo de reducción en la época toledana) y las comunidades campesinas en sus alrededores, en la década de los años noventa. Por varias razones histórico-sociales, existe un trilingüismo marcado en esta zona de Bolivia. Es un tema que Albó también ha estudiado, en publicaciones con Olivia Harris; por ejemplo (Harris y Albó 1986).

Como sabemos, el quechua se extendió al territorio del Alto Perú bajo el incario, pero mayormente a los valles templados alrededor de Cochabamba. La zona del Norte de Potosí fue aimarahablante, al igual que el altiplano boliviano, hasta mediados del siglo XX. Siguiendo a la reforma agraria de 1953 y el ascenso de la minería del estaño en la región durante las décadas de 1960 y 1970, hubo un flujo de trabajadores quechuahablates del valle de Cochabamba que se quedaron a trabajar en el sector agrario del altiplano después de la caída de la industria minera y los cambios en la economía política de los años ochenta y noventa (Harris y Albó 1986). El movimiento migratorio del quechua de los valles hacia la zona aimarahablante del altiplano por razones económicas asentó la influencia del aimara sobre el quechua, como observamos hasta el día de hoy. También hay que tomar en cuenta el funcionamiento de los aillus tradicionales que, con su configuración de archipiélago, fomentaban el matrimonio y el trueque entre aimarahablantes de las alturas con quechuahablantes de los valles, generando redes sociales intrafamiliares aimara-quechuas. El castellano hablado se conocía mayormente entre los

\footnotetext{
2 Sobre la sintaxis del quechua de Cochabamba, el trabajo de Yolanda Lastra (1968) precedió al de Albó. Una forma mixta de quechua con castellano se encuentra socialmente extendida en la zona urbana de Cochabamba, denominada "quechuañol" en el habla popular. El libro de Inge Sichra $(2003,115-116)$ sobre lengua y sociedad en dos provincias de Cochabamba ofrece una descripción de algunos rasgos sintácticos y morfosintácticos del quechuañol. Sichra también discute las marcadas influencias del estrato quechua en la gramática del castellano popular de Cochabamba (112-114; cf. Howard 2007, 162). El estudio de Babel (2018) trata el contacto lingüístico-sociocultural en la zona valluna entre Cochabamba y Santa Cruz. La relación entre las lenguas quechua y aymara en su extensión histórica-lingüística es tratada en Cerrón-Palomino (2008).
} 
hombres, aunque con la mejor oferta educativa desde mediados de los noventa, las mujeres jóvenes también dominan esa lengua.

Así que, en la época en la cual efectué este estudio, el panorama sociolingüístico de la zona valluna de San Pedro de Buenavista se presentaba como sigue: bilingüismo aimara-quechua entre los mayores; trilingüismo con castellano entre los de treinta años para arriba; bilingüismo quechuacastellano entre los jóvenes. Es importante anotar que el aimara, en este contexto socio-geográfico, era la lengua de menor prestigio; la gente del campo tendía a ocultar el hecho de que hablaba el aimara frente a la gente del pueblo. Su competencia en esta lengua se revelaba cuando ya sentían confianza, y solamente en contextos familiares o rituales, como veremos. Esto contrasta con la situación en los departamentos de tierras altas como Oruro, La Paz y, por el lado del Perú, Puno, donde el aimara mantiene vigor y un cierto prestigio social.

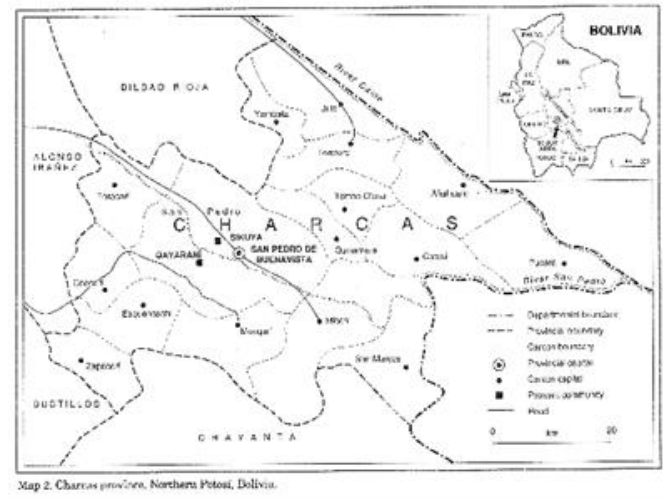

Figura 1: Ubicación de la provincia de Charcas en Bolivia Fuente: Howard-Malverde 1995, 148

Algunas cifras de los censos de población en Bolivia nos demuestran las tendencias de substitución lingüística a favor del castellano durante las últimas cinco décadas (Cuadro 1). Entre los censos del año 1976 y 1992, el monolingüismo castellano había subido en un $24,9 \%$, mientras que el monolingüismo quechua y aimara había decrecido en un $37,7 \%$ y $51,5 \%$, respectivamente (República de Bolivia 1993, citado en Howard-Malverde 1995, 144-145). Sin embargo, de acuerdo con el censo de 1992, época en la cual se 
llevó a cabo el presente estudio, a nivel nacional el departamento de Potosí tenía el porcentaje de castellanohablantes más bajo (68,5\%), el porcentaje de quechuahablantes más elevado $(83,1 \%)$ y la proporción de aimarahablantes en tercera posición después de los departamentos de Oruro y La Paz $(10,7 \%)$ (Censo 1993, citado en Howard-Malverde 1995, 146). Estas cifras no suman el cien por cien por la alta incidencia de bilingüismo y en algunos casos trilingüismo.

\begin{tabular}{|l|c|c|l|c|l|l|}
\hline Año de censo & \multicolumn{3}{c}{1976} & \multicolumn{5}{c|}{1992 (> 6 años) } & \multicolumn{2}{c|}{2001 (>6 años) } \\
\hline Lengua & $1000 s$ & $\%$ & $1000 s$ & $\%$ & $1000 s$ & $\%$ \\
\hline Castellano & $3.210,0$ & 78,8 & $4.594,1$ & 87,4 & $6.097,1$ & 87,7 \\
\hline Quechua & $1.594,0$ & 39,7 & $1.085,8$ & 34,3 & $2.124,0$ & 30,6 \\
\hline Aimara & $1.156,0$ & 29,8 & $1.237,7$ & 23 & $1.462,3$ & 21,0 \\
\hline $\begin{array}{l}\text { Guaraní y otras } \\
\text { lenguas nativas }\end{array}$ & 56,0 & 1,1 & 129,6 & 2,5 & 101,2 & 1,5 \\
\hline $\begin{array}{l}\text { Lenguas } \\
\text { extranjeras }\end{array}$ & - & - & 118,2 & 2,2 & 241,4 & 3,4 \\
\hline Población total & $4.613,4$ & 100 & $7.132,0$ & 100 & $8.261,2$ & 100 \\
\hline Total > 6 años & - & - & $5.256,3$ & - & $6.948,6$ & - \\
\hline
\end{tabular}

Cuadro 1: Bolivia: Evolución sociolingüística de la población 1976-2001 Fuente: Molina y Albó 2006

Estas cifras basadas en tres censos demuestran una relativa estabilidad en el número de hablantes de quechua y aimara durante las décadas recientes, con un porcentaje creciente de bilingüismo con el castellano. Para actualizar estas cifras con referencia al censo del 2012, este reporta una proporción de $17,15 \%$ de quechuahablantes respecto de la población total (Andrade y Howard, en prensa).

La pregunta que se hizo fue sobre pertenencia étnica. Sobre una población total de 10.027.254 personas hubo 6.916 .732 que se declararon de "pertenencia étnica" (más de dos tercios); de ellos, 1.281.116 se decían quechuas; 1.191 .352 se decían aimaras. Sin embargo, declararse étnicamente quechua o aimara no equivale necesariamente a ser hablante de la lengua. 
En el Norte de Potosí, en los centros urbanos como el pueblo de San Pedro de Buenavista (Fig. 2) en los años 1990, se había consolidado un bilingüismo castellano-quechua estable. Los sanpedrinos migran sobre todo a Cochabamba y La Paz, donde el castellano prevalece, pero cuando regresan al pueblo en época de cosecha o de fiestas, rápidamente retoman su bilingüismo provincial. El quechua no lleva estigma en el medio urbano norpotosino, al contrario; la lealtad hacia el quechua es fuerte entre los pueblerinos bilingües.

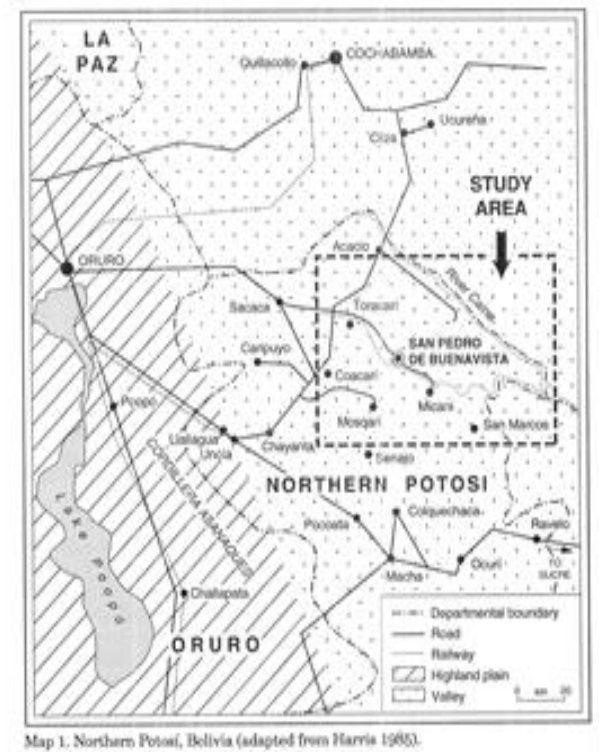

Figura 2: Ubicación del sitio del estudio

Fuente: adaptado de Harris (1985)

En la figura 3 se ilustra esquemáticamente la distribución de las tres lenguas en el espacio geográfico y social: el quechua está situado en una posición intermedia entre el aimara de la periferia rural y el castellano del centro urbano. Los símbolos indican: + presencia de la lengua; - ausencia de la lengua; +/- presencia dominante de la lengua; -/+ presencia subordinada de la lengua. 


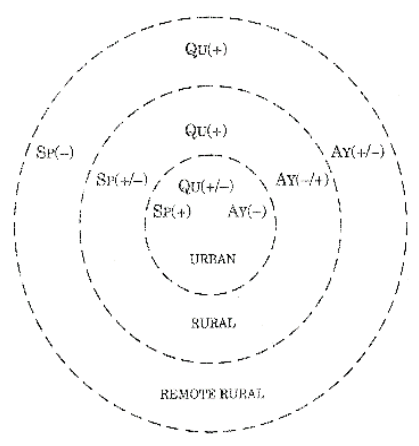

Figura 3: Modelo de contacto aimara-quechua-castellano en el valle de San Pedro de Buenavista

Fuente: Howard-Malverde $(1995,142)$

En este estudio se combina el análisis sociolingüístico a nivel macro con los datos lingüísticos a nivel micro; se busca interpretar los datos formales (el léxico, particularmente) a la luz del contexto sociocultural. Los rasgos de contacto lingüístico que vamos a examinar demuestran que el aimara está en una relación de "sustrato" relativo al quechua en términos directos, y de "sustrato" relativo al castellano en términos más indirectos; es decir que las huellas del aimara que se encuentran en el castellano muchas veces tendrán su origen en un quechua aimarizado, y no en el contacto directo entre el aimara y el castellano.

El trabajo de campo se llevó a cabo, por una parte, en el pueblo de San Pedro de Buenavista, población en esa época de unas mil personas, y, por otra parte, en las comunidades campesinas de Sikuya (margen oriental del río) y Qayarani (margen occidental del rio). La ubicación geográfica de las dos comunidades incide mucho en su carácter sociolingüístico. En Sikuya era menos evidente que se sabía aimara, los habitantes eran predominantemente quechuahablantes, los hombres más jóvenes dominaban también el castellano. En Qayarani, aunque ostensiblemente el quechua era la lengua predominante, con menos castellano, después de ganar familiaridad con los comuneros tras varias visitas de retorno, pude darme cuenta de que el aimara se hablaba extensivamente, incluso entre los jóvenes, pero que se lo guardaban para conversaciones íntimas, en contextos familiares, en los ritos, y lejos de la 
presencia de personajes oficiales como la maestra de la escuela. ${ }^{3}$ En suma, el aimara se había convertido en una lengua de comunicación intracomunal no utilizada en contextos formales y efectivamente invisible frente a los pueblerinos (que decían frecuentemente que "no se habla aimara por aquí") y frente a los planificadores educativos, que solamente buscaban reforzar el uso del quechua en las escuelas y no el aimara.

El interés de la articulación entre las tres lenguas en esta región de Bolivia se hizo patente para mí por primera vez en el curso de una conversación que tuve con una señora de la comunidad de Sikuya, quechua monolingüe de unos treinta y cinco años de edad. Le pregunté si en su comunidad se sabía hacer ofrendas a la Pachamama en el mes de agosto. Estábamos conversando en quechua y utilicé esa palabra: "Pachamama". Su respuesta fue la siguiente: “'Pachamama' ninku castellanomanta, 'pachamama' ninchik. Kay qhichwamantarí 'wirjina'. 'Wirjina' a 'wirjina'” [“Dicen 'pachamama' en castellano, decimos 'pachamama'. Y en quechua es 'wirjina'. 'Wirjina' ya, 'wirjina'”]. (Howard-Malverde 1995, 143)

Voy a comentar tres puntos con respecto a la aclaración de esta señora. Primero, el juego de prácticas y creencias religiosas a las cuales alude al utilizar la palabra "wirjina" no tienen nada que ver con el culto a la Virgen de la iglesia católica, y tienen mucho que ver con el culto a la tierra que se practica en paralelo con el cristianismo. La palabra "wirjina" no es indicio del sincretismo religioso. Su referente es la tierra animada; su uso en este contexto es, por lo tanto, un ejemplo de "remodelación vernácula" (Hill \& Coombs 1982), es decir, el significado que tenía en la lengua de origen ha sido cambiado en su traslado a otro contexto referencial. Segundo, los bilingües urbanos utilizan la palabra "pachamama" para referirse a la madre tierra -la palabra "wirjina" les es desconocida- y también le hacen ofrendas, con sacrificios de animales, en los meses de febrero y agosto. Utilizan la palabra "Virgen" para referirse a la Virgen María del culto católico. Tercero, la pronunciación "wirjina" en boca de los quechuahablantes del campo manifiesta reglas de refonologización que se aplican en aimara, no en quechua, a las palabras de origen castellano; en particular, la adición de una vocal final a las palabras que terminan en consonante. En resumidas cuentas, la palabra "wirjina" es un hispanismo resemantizado llegado al quechua del Norte de Potosí mediante el conducto

\footnotetext{
${ }^{3}$ Durante el trabajo de campo en 1993, me acompañó el antropólogo Andrew Canessa, hablante de aimara. Sus interacciones con los "campo runa" nos revelaron la extensión del aimara en el repertorio lingüístico.
} 
del aimara y no directamente desde el castellano. Volveré a este ejemplo sugerente hacia el final del artículo.

En cuanto a mi metodología y objetivos, hice varias visitas a la zona con el fin de estudiar la lengua y la cultura del norte potosino; compilé un corpus de transcripciones basado en unas treinta horas de conversaciones y entrevistas grabadas en audio y video con la gente del lugar. Este material sirvió de insumo para la confección de un programa multimedia para la enseñanza del quechua como lengua extranjera (Howard et al. 2014). Al analizar las transcripciones, hice un estudio de los rasgos de contacto lingüístico aimara-quechuacastellano que tipifica el habla quechua de la zona, por un lado, entre los hablantes del pueblo y, por otro lado, entre los comuneros (estos grupos se autodenominan en quechua llaqta runa [gente del pueblo] y campo runa [gente del campo] respectivamente.

Encontré rasgos tanto fonológicos como léxicos que me parecían dignos de una encuesta entre los hablantes, con dos objetivos: (i) determinar en qué medida los hablantes estaban conscientes del contacto lingüístico que caracterizaba su habla, y la importancia que atribuían a tal fenómeno; (ii) indagar sobre la relación del léxico - de origen mixto entre quechua, aimara y castellano- con la estructura social y las otras prácticas culturales. Apliqué la encuesta a un total de siete personas. Fue una muestra pequeña pero bien estratificada, pues incluía tanto personas del pueblo (castellano/quechua), como personas de Sikuya (más quechua/poco castellano/poco aimara) y Qayarani (más quechua/un poco más aimara/poco castellano), tanto hombres como mujeres, de una cierta edad y más jóvenes. Por el hecho de que basé la encuesta en un corpus previamente analizado del habla de unas cuarenta personas provenientes de los mismos tres contextos sociolingüísticos, estimo que los resultados reflejan los usos fonológicos y léxicos tal como estos se manifiestan de manera general en el valle de San Pedro.

En cuanto a los efectos de contacto a nivel fonológico, solamente voy a comentar el aspecto de la refonologización de las palabras de origen castellano (o "préstamos") al ser pronunciadas en quechua y aimara. La refonologización es más frecuente y más arraigada en el quechua de los campo runa que en el de los Ilaqta runa. ${ }^{4}$ En el quechua rural, un rasgo que

\footnotetext{
${ }^{4}$ El quechua de los llaqta runa de San Pedro, de acuerdo con lo que ellos mismos me decían, tiene más influencia en general del castellano. Habría que investigar en qué medida se aproxima al quechua bastante mezclado con el castellano que se habla en la ciudad de Cochabamba, tal como ha sido descrito por Albó (1974).
} 
sobresale es la adición de una vocal final, como ocurre con la ya mencionada palabra "Virgen", que se realiza [wirjina]. Otros ejemplos incluyen [jawasa] de "habas", [limuna] de "limón" y [jawuna] de "jabón". Podemos suponer que este patrón de refonologización se debe a la influencia aimara, por el hecho de que en esta lengua los sustantivos terminan sistemáticamente en vocal. Cualquier palabra de origen castellano que termina en consonante se modifica de esta manera al ser incorporada al aimara (Cuadro 2). Así que parece poco discutible que los cambios en los hispanismos aquí comentados, de "jabón" a [jawuna], etc., ocurrieron en el aimara antes de pasar al quechua, durante el período de intensa colonización del aimara por el quechua ocurrida en la región durante la segunda mitad del siglo XX.

\begin{tabular}{|c|c|c|c|}
\hline $\begin{array}{c}\text { Nivel de Competencia } \\
\text { Lingüística }\end{array}$ & $\mathrm{ai}-/ q u+/$ cast+ & $\mathrm{ai}+/ \mathrm{qu}+/$ cast $(+)$ & $\mathrm{ai}+/ q u+/$ cast- \\
\hline \multicolumn{4}{|l|}{$/ d /[d>t>r]$} \\
\hline Día & [diya] & [tiya] & [riya] \\
\hline Cordero & [kordero] & [kurdiru] & [kurtiru] \\
\hline \multicolumn{4}{|l|}{$/ b /[b>w]$} \\
\hline Cebada & [sebada] & [sibada] & [siwara] \\
\hline Cebolla & [sebolla] & [sibolla] & [siwilla] \\
\hline Alverja & [arbexa] & [albirxa] & [alwirixa] \\
\hline Oveja & [obexa] & [obixa] & [uwixa] \\
\hline \multicolumn{4}{|c|}{ /g/ [g > x] más expansión silábica } \\
\hline Trigo & [trigu] & [trigu] & [tirixu] \\
\hline \multicolumn{4}{|c|}{ Adición de vocal final } \\
\hline Habas & [abas] & [abas] & [xawasa] \\
\hline Limón & [limon] & [limun] & [limuna] \\
\hline Jabón & [xawun] & [xawuna] & [xawuna] \\
\hline Virgen & [birxen] & [wirxina] & [wirxina] \\
\hline Mandil & [mandil] & [mantil] & [mantila] \\
\hline Costal & [kostala] & [kostala] & [kustala] \\
\hline
\end{tabular}

Cuadro 2: Refonologización de los hispanismos en el quechua de San Pedro de Buenavista 
Con respecto al contacto lingüístico a nivel del léxico, me enfoco en las influencias en el quechua que, en esta región, provienen, por un lado, del aimara y, por otro, del castellano. En cuanto a la relación entre el aimara y el quechua, de acuerdo con Willem Adelaar (1986), se estima que estas lenguas comparten sus recursos léxicos en un $25 \%$ a $30 \%$. Además de este fondo general compartido, ocurren lo que Adelaar denomina los "préstamos incidentales" (incidental lexical borrowing) en ciertas variantes, de extensión restringida a una localidad. A este fenómeno "incidental" se puede atribuir la presencia de algunos de los elementos aimaras en el léxico quechua del Norte de Potosí.

Siguiendo las pautas identificadas por Appel y Muysken (1987), la influencia del léxico castellano en las lenguas indígenas latinoamericanas tiene, desde el punto de vista teórico, una gama de efectos y funciones, entre ellos:

- Reemplazar totalmente el lexema indígena.

- Llenar un vacío en el que la lengua indígena, por razones culturales, carece de recursos.

- Causar un cambio (muchas veces, una retracción) en el campo semántico de la palabra indígena.

- Coexistir con el lexema indígena, constituyendo una alternativa (sinónimo).

- Adquirir un nuevo significado en el nuevo medio ambiente lingüístico; una "remodelación vernácula" ("vernacular remodelling", de acuerdo a Hill y Coombs, 1982).

En cuanto al estudio del léxico, mi procedimiento al preparar la encuesta fue clasificar las palabras por campos semánticos tal como el corpus lo sugería: palabras de léxico general, términos de parentesco, vestimenta, elaboración de la chicha, léxico del tejido, vocabulario ritual.

\section{Léxico general}

En cuanto al quechua, en muchos casos se había introducido una palabra de origen castellano que duplicaba o compartía de alguna forma la función y el significado de la palabra propiamente quechua, en los términos arriba enumerados. Tratándose de los entrevistados urbanos, ellos reconocían el origen castellano, por ejemplo, de bañakuy, escribiy, etc., pero no se discernía entre ellos en ningún caso reflexiones de tipo purista (Cuadro 3). Nadie decía que mezclar el quechua con el castellano era problemático; 
incluso, al contrario, una entrevistada del pueblo hizo la observación de que "aquí hablamos el quechua un poco refinado, ¿no ves que mezclamos con castellano?". Cuando les pedí alguna información sobre el equivalente en lengua aimara, nadie podía responder. Era frecuente escuchar a los sanpedrinos urbanos decir que "no hay aimara por aqui".

\begin{tabular}{|l|l|l|}
\hline Lexema Quechua & Lexema Aimara & Glosa Castellana \\
\hline tumay/ukyay & Umaña & Beber \\
\hline trabajay/llank'ay & Irnaqaña & Trabajar \\
\hline descansay/samarikuy & samart'aña & Descansar \\
\hline bañakuy/mayllakuy & Jarisiña & Lavarse \\
\hline escribiy/qillqay & Qillqaña & Escribir \\
\hline
\end{tabular}

\section{Cuadro 3: Vocabulario general (ejemplos seleccionados)}

En el grupo de los entrevistados rurales no había el tipo de reflexión metalingüística que se encontraba en el pueblo. es decir, no se analizaban los vocablos en términos de su "origen" en tal o cual lengua; se aceptaban ambas palabras como "quechua" y "castellano" con comentarios como "se utilizan en ambas lenguas". Cuando les pedí un equivalente en aimara, a pesar de que los entrevistados rurales usaban poco esa lengua en sus vidas cotidianas, por lo que pude observar, me daban la traducción al aimara sin ningún problema. Estaba claro que el aimara era un componente relativamente pasivo de su repertorio lingüístico, pero en absoluto estaba ausente, en contraste con lo que solían afirmar los habitantes urbanos. El otro punto que llamó la atención fue que cuando pregunté si el hispanismo que se presentaba como una alternativa a los lexemas quechuas también se podría utilizar en el aimara, me dijeron que no. Las palabras formalmente reconocibles como préstamos del castellano (desde nuestro punto de vista) se aceptaban como palabras que servían en el quechua ("valen para las dos lenguas"), pero no para un contexto aimara.

Debido al hecho de que el idioma aimara tiene poca vitalidad en los valles de San Pedro, era más sorprendente que se admitieran escasamente los préstamos del castellano. De hecho, el vocabulario del aimara que elicité en las entrevistas era más "puro" que el quechua, a pesar de que el aimara era la 
lengua más amenazada. Los lexemas refonologizados que acabo de mencionar no eran reconocidos como hispanismos; la misma refonologización los aimariza. El aimara ha sido el conducto para que palabras como jawasa, tiriju, siwara, jawuna y alwirija manifiesten esta forma en el quechua de San Pedro. Desde cierto punto de vista, estos "hispanismos" deben considerarse "aimarismos" una vez que pasan al léxico quechua; es decir, son rasgos de estrato aimara en primera instancia y no de estrato castellano. Además, su etimología no es ni evidente ni pertinente para los hablantes.

Esta situación es un reflejo de que el desplazamiento lingüístico desde el aimara por el castellano en esta región ha pasado por una etapa intermedia de desplazamiento del aimara por el quechua. El quechua, ya con sus influencias aimaras, ha sido la lengua que entró directamente en contacto con el castellano. Además, cabe aclarar, el contacto entre el quechua y el castellano ha sido un fenómeno urbano en su primera etapa. El quechua castellanizado se expande en un proceso ulterior hacia las comunidades más remotas donde sobrevive el aimara. En estos valles del norte potosino, el aimara y el castellano no han sido lenguas que estuvieran tan directamente en contacto, a diferencia de lo que ocurre en las tierras altiplánicas de Oruro, La Paz y las orillas del Lago Titicaca, donde, además, el aimara es una lengua tanto de la ciudad como del campo.

\section{Los términos de parentesco}

Los términos de parentesco y afinidad pertenecen a un campo semántico ligado a la conceptualización de la estructura y las relaciones sociales. En una situación de colonización, cuando estas estructuras y relaciones son sometidas a cambios, la pregunta es en qué medida la nomenclatura puede resistir. En los datos del Norte de Potosí hay diferencias notables entre el léxico aimara y quechua del campo, por un lado, y entre el léxico quechua del campo y el del pueblo, por otro. Comentaremos algunos ejemplos (Cuadro 4).

Los términos de parentesco y de afinidad aimara fueron elicitados de hablantes bilingües aimara-quechua en el contexto rural. El vocabulario aimara manifiesta poca interferencia de las otras dos lenguas. Los únicos hispanismos son "kuñadu" (hermano del esposo), "kuñada" (hermana de la esposa), "awila" (madre de la mamá o del papá) y "tata" (padre y hermano del padre). Los lexemas compartidos entre quechua y aimara son cuatro. Tomando en cuenta que el aimara ya era poco utilizado activamente y que los pueblerinos negaban su existencia en el valle, estos datos son llamativos. 


\begin{tabular}{|c|c|c|c|c|}
\hline Aimara & Quechua & $\begin{array}{l}\text { Aimara y } \\
\text { Quechua }\end{array}$ & Castellano & Significado \\
\hline achachi & (ninguno) & & & $\begin{array}{l}\text { padre de madre, } \\
\text { padre de padre }\end{array}$ \\
\hline laqusï & & & & $\begin{array}{l}\text { esposa del hermano } \\
\text { de madre }\end{array}$ \\
\hline lari & & & & $\begin{array}{l}\text { hermano de esposa, } \\
\text { hermano de madre }\end{array}$ \\
\hline chacha & & & & esposo \\
\hline tullqa & & & & $\begin{array}{l}\text { esposo de hermana, } \\
\text { esposo de hija }\end{array}$ \\
\hline yuqch'a & & & & $\begin{array}{l}\text { esposa de hermano, } \\
\text { esposa de hijo }\end{array}$ \\
\hline jila & & & & hermano \\
\hline kullaka & & & & hermana \\
\hline \multirow[t]{9}{*}{ allchhi } & & & & $\begin{array}{l}\text { hija de hijo, hija de } \\
\text { hija, hijo de hijo, hijo } \\
\text { de hija }\end{array}$ \\
\hline & & mama & & madre \\
\hline & & warmi & & esposa \\
\hline & & wawa & & hijo, hija \\
\hline & & Ipa(la) & & $\begin{array}{l}\text { hermana de esposo, } \\
\text { hermana de padre }\end{array}$ \\
\hline & & & awila & $\begin{array}{l}\text { madre de madre, } \\
\text { madre de padre }\end{array}$ \\
\hline & & & kuñadu & hermano de esposo \\
\hline & & & kuñada & hermana de esposa \\
\hline & & & tata & $\begin{array}{l}\text { padre, hermano de } \\
\text { padre }\end{array}$ \\
\hline
\end{tabular}

Cuadro 4: Lengua fuente para los términos de parentesco en aimara

En el caso de la terminología quechua de parentesco y de afinidad entre los campo runa, el caso es el contrario. De los dieciséis términos registrados, nueve son hispanismos, tres se originan en el aimara y solamente uno (qusa "marido") puede decirse de origen netamente quechua (Cuadro 5). Reitero que estas observaciones etimológicas se basan en criterios formales de lingüista; para los hablantes mismos, todos son considerados como quechuas. Algunos son reconocidos como compartidos entre lenguas: 
"ermanu"/"ermana" son tanto quechuas como castellanos; "tullqa" y "yuqch'a" son compartidos entre quechua y aimara (sus equivalentes en castellano son cuñado/yerno o cuñada/yerna). Los vocablos aimara "lari" (hermano de la esposa o de la madre) e "ipala" (hermana del esposo o del padre), son glosados por "tiyu" y "tiya" en el quechua rural (son ejemplos de palabras de origen español cuyo significado es alterado en el nuevo medio ambiente lingüístico).

\begin{tabular}{|c|c|c|c|c|}
\hline Aimara & Quechua & $\begin{array}{l}\text { Aimara y } \\
\text { Quechua }\end{array}$ & Castellano & Significado \\
\hline tullqa & & & & $\begin{array}{l}\text { esposo de hermana, } \\
\text { esposo de hija }\end{array}$ \\
\hline yuqch'a & & & & $\begin{array}{l}\text { esposa de hermano, } \\
\text { esposa de hijo }\end{array}$ \\
\hline \multirow[t]{14}{*}{ allchhi } & & & & $\begin{array}{l}\text { hija de hijo, hija de } \\
\text { hija, hijo de hijo, hijo } \\
\text { de hija }\end{array}$ \\
\hline & qusa & & & Esposo \\
\hline & & mama & & Madre \\
\hline & & warmi & & Esposa \\
\hline & & wawa & & hijo, hija \\
\hline & & & awilu & $\begin{array}{l}\text { padre de padre, } \\
\text { padre de madre }\end{array}$ \\
\hline & & & awila & $\begin{array}{l}\text { madre de padre, } \\
\text { madre de madre }\end{array}$ \\
\hline & & & tiyu & $\begin{array}{l}\text { hermano de esposa, } \\
\text { hermano de madre }\end{array}$ \\
\hline & & & tiya & $\begin{array}{l}\text { hermana de esposo, } \\
\text { hermana de padre, } \\
\text { hermana de madre }\end{array}$ \\
\hline & & & ermanu & hermano \\
\hline & & & ermana & hermana \\
\hline & & & kuñadu & hermano de esposo \\
\hline & & & kuñada & hermana de esposa \\
\hline & & & tata & $\begin{array}{l}\text { padre, hermano de } \\
\text { padre }\end{array}$ \\
\hline
\end{tabular}

Cuadro 5: Lengua fuente para los términos de parentesco en quechua 
En el Norte de Potosí, "tullqa" (cuñado y yerno) y "yuqch'a" (cuñada y nuera), utilizados tanto en aimara como en quechua, se pueden considerar como rasgos de sustrato aimara con relación al quechua. Sus equivalentes en el quechua sureño qatay (yerno) y qachun (nuera) no se escuchan en esta región. Tullqa y yuqch'a se refieren a una categoría bi-generacional de "tomador de esposa" (spouse-taker) en el sistema de afinidad de los campo runa, una perspectiva que no prevalece entre los llaqta runa, donde estos términos son sustituidos por hispanismos (yerno/yerna/ kuñado/kuñada) o, si son utilizados, su campo semántico es restringido a yerno/yerna.

La terminología de parentesco nos provee un ejemplo del efecto del contacto lingüístico entre dos marcos de referencia divergentes en cuanto a la estructura social (Collins 1983). En el punto de divergencia (el yerno y el cuñado ocupan espacios semánticos distintos en el pensamiento de los llaqta runa, y similares en el de los campo runa), el léxico quechua se torna inadecuado en el espacio urbano, y se acude a los hispanismos para expresar las categorías propias de la cultura de origen europeo. Estos hispanismos poco a poco van infiltrando el quechua del campo, a partir del quechua de la urbe; solamente las categorías más arraigadas como las de tullqa y yuqch'a se resisten a la hispanización en este campo léxico.

\section{Vocabulario de la vestimenta}

La vestimenta tradicional en el Norte de Potosí, como en todas partes de los Andes, es una señal externa de la identidad, a la par con la lengua misma. Hemos registrado catorce lexemas para referirse a la vestimenta tradicional: seis de estos considerados compartidos entre el aimara y el quechua, y cuatro especializados en cada lengua (chumpi, Iliklla, aqsu, ch'uspa en quechua). En el pueblo, en varios casos los bilingües castellano-quechua no sabían expresar la palabra quechua en castellano; simplemente usaban el quechuismo con pronunciación castellana (chuspa, chulu). En otros casos, declararon que "no se conoce en el pueblo, no se puede decir" (el caso de phanta, aqsu, tullma). Las palabras chakita y wujanta son hispanismos (de "chaqueta" y "bufanda") que cambian de función cultural y, por tanto, de contenido semántico en su nuevo entorno: la chaqueta bordada y la faja colgante del cinturón son prendas masculinas ambas (Cuadro 6). 


\begin{tabular}{|c|c|c|}
\hline Léxico Compartido & \multicolumn{2}{|c|}{ Significado } \\
\hline almilla (< almilla) & \multicolumn{2}{|c|}{ vestido de bayeta (de mujer) } \\
\hline tullma & \multicolumn{2}{|c|}{ cordel para amarrar el pelo (de mujer) } \\
\hline phanta & \multicolumn{2}{|c|}{ paño para cubrir la cabeza (de mujer) } \\
\hline inkhuña & \multicolumn{2}{|c|}{ paño para envolver comida o coca } \\
\hline phullu & \multicolumn{2}{|c|}{ frazada tejida en telar } \\
\hline ch'ullu & \multicolumn{2}{|c|}{ gorro tejido en palos, chullo (de hombre) } \\
\hline chakita (< chaqueta) & \multicolumn{2}{|c|}{ chaqueta de bayeta bordada (de hombre) } \\
\hline wujanta (< bufanda) & \multicolumn{2}{|c|}{ faja ancha tejida en telar (de hombre) } \\
\hline \multicolumn{2}{|c|}{ Léxico Divergente } & Significado \\
\hline aimara & quechua & \\
\hline t'isnu/wak'a & chumpi & faja angosta tejida en telar \\
\hline awayu & Tliklla & $\begin{array}{l}\text { rebozo en forma cuadrada } \\
\text { tejido en telar }\end{array}$ \\
\hline urkhu & aqsu & $\begin{array}{l}\text { pieza tejida en telar; se lleva } \\
\text { colgada de la cintura atrás } \\
\text { (de la mujer) }\end{array}$ \\
\hline wallqipu & ch'uspa & bolsa para llevar coca \\
\hline
\end{tabular}

Cuadro 6: Terminología de la vestimenta en aimara y quechua

\section{Terminología para la elaboración de la chicha}

Entre el léxico que pertenece al campo de la elaboración de la chicha y el que pertenece al arte del tejido, hay una diferencia notable por el grado de especialización léxica que se manifiesta en uno y otro. Mientras que en el vocabulario del tejido hay un alto grado de especialización (como veremos), en el vocabulario de la chicha solo los vocablos para maíz y chicha son especializados ('maíz' Qu. sara v. Ay. tunqu y 'chicha' Qu. aqha v. Ay. k'usa); los demás lexemas en este campo son compartidos. A un hombre aimara-quechua bilingüe se le pidió relatar el proceso de elaborar la chicha en las dos lenguas consecutivamente. Al hacerlo, dijo que era necesario utilizar el vocabulario quechua para hablar de este tema, aunque fuera por medio del aimara. El aimara, según él, no tenía el vocabulario necesario. Esta restricción es probablemente el reflejo de que la chicha se produce mayormente en los valles templados donde se cultiva el maíz e, históricamente por lo menos, se habla quechua de manera predominante. Cuando se entrevistó a los bilingües castellano-quechua del pueblo, muy poco sabían traducir este léxico al castellano. Como la elaboración de la chicha es una actividad tanto urbana 
como rural, los llaqta runa conocían perfectamente bien su terminología. Para hablar en castellano del tema, recurrían al vocabulario quechua, por ejemplo, la frase quechua chuwa qunchu para referirse al líquido que se decanta de la sedimentación final, se tradujo al castellano con una paráfrasis y con lexemas tomados del quechua: "es el primer upi que sale del janchi" (Cuadro 7).

\begin{tabular}{|c|c|c|}
\hline Léxico Compartido & \multicolumn{2}{|c|}{ Significado } \\
\hline wiñapu & \multicolumn{2}{|c|}{ granos de maíz brotados } \\
\hline qhaqu o muk'u & \multicolumn{2}{|c|}{ pasta de maíz } \\
\hline upi & \multicolumn{2}{|c|}{ primera decantación } \\
\hline q'ita & \multicolumn{2}{|l|}{ Melaza } \\
\hline janch'i o qunchu & \multicolumn{2}{|c|}{ sedimento final } \\
\hline chuwa qunchu & \multicolumn{2}{|c|}{ decantación del sedimento final } \\
\hline p'uñu & \multicolumn{2}{|c|}{ jarrón de barro para fermentación } \\
\hline wirkhi & \multicolumn{2}{|c|}{ jarra para decantación } \\
\hline \multicolumn{2}{|c|}{ Léxico Divergente } & Significado \\
\hline aimara & Quechua & \\
\hline tunqu & Sara & maíz \\
\hline k'usu & Aqha & chicha \\
\hline
\end{tabular}

Cuadro 7: Terminología de elaboración de la chicha en aimara y quechua

\section{Terminología del tejido}

En el caso de la terminología del tejido, hay un mayor grado de especialización léxica que en el de la chicha. Así y todo, once de los dieciséis términos identificados son compartidos (Cuadro 8). Los vocablos específicos de una lengua son los que se refieren a conceptos menos técnicos. El tejido es un arte tan propio de los sectores aimara como quechuahablantes y de las zonas ecológicas que habitan tradicionalmente. El castellano, además, posee más recursos léxicos para hablar de técnicas textiles que en el caso de la fabricación de la chicha. Solo cuando las técnicas divergen de las occidentales había dificultad. Por ejemplo, los palos verticales del telar andino, llamados aykata, no encuentran glosa en castellano; el bilingüe utiliza la palabra quechua en ambas lenguas. Lo mismo vale para la palabra khallu para hablar de la mitad de la prenda tejida, que se debe unir a su otra mitad con una costura después de tejer (por ejemplo, en una lliklla o un poncho). Solamente se podía glosar "khallu" con una paráfrasis: "un lado de la tapa". 
Es interesante notar que, al conversar sobre el vocabulario del tejido con los pueblerinos, muchas veces daban explicaciones en tercera persona del plural, distanciándose de las prácticas en cuestión. El tejido es un arte propio "de ellos". De hecho, no se practica entre los Ilaqta runa; más bien, al contrario, se manda a tejer los artículos requeridos con tejedores y tejedoras del campo, que suelen venir de sus casas al pueblo para prestar el servicio. El léxico del tejido es menos propio de los bilingües urbanos que el léxico de la chicha, por estas razones socioeconómicas y culturales.

\begin{tabular}{|c|c|c|}
\hline \multicolumn{2}{|c|}{ Léxico Compartido } & Significado \\
\hline \multicolumn{2}{|l|}{ K'antiy } & torcelar hilos \\
\hline \multicolumn{2}{|l|}{ Khiwiy } & ovillar hilos \\
\hline \multicolumn{2}{|l|}{ Mismiy } & torcelar hilos en huso \\
\hline \multicolumn{2}{|l|}{ Phariy } & hilar con poca torsión \\
\hline \multicolumn{2}{|l|}{ wich'uy } & golpear los hilos de la trama \\
\hline \multicolumn{2}{|l|}{ Ayka } & marco vertical del telar \\
\hline \multicolumn{2}{|l|}{ Illawa } & Tizo \\
\hline \multicolumn{2}{|l|}{ Huñi } & madeja \\
\hline \multicolumn{2}{|l|}{ Khallu } & una mitad de la pieza tejida 5 \\
\hline \multicolumn{2}{|l|}{ Khata } & calada $^{6}$ \\
\hline \multicolumn{2}{|l|}{ Wichkhata } & $\begin{array}{l}\text { palo para prensar la tela entre las } \\
\text { urdimbres }\end{array}$ \\
\hline \multicolumn{2}{|c|}{ Léxico Divergente } & Significado \\
\hline Aimara & quechua & \\
\hline Sawuña & away & tejer \\
\hline Qapusiña & phuchkhay & hilar fibra con rueca \\
\hline t'arwa & millma & fibra natural \\
\hline Qïpa & $\operatorname{mini}$ & trama \\
\hline ch'anka & q'aytu & hilo de fibra natural \\
\hline
\end{tabular}

Cuadro 8: Terminología del tejido en aimara y quechua7

\footnotetext{
5 "Mä khallu" o "mankhallu" sería una mitad de un awayo o poncho en otras variedades dialectales (Arnold, p.c. 18/07/19).

${ }^{6}$ El espacio que separa los hilos de la urdimbre en la acción de tejer

${ }^{7}$ El trabajo de Yapita, Arnold y Espejo (2014) fue imprescindible para aclarar el significado de los términos aimaras.
} 


\section{El vocabulario ritual: el lenguaje de las "ch'allas"}

En el campo semántico de los ritos, la lengua aimara ha sido más tenaz en el Norte de Potosí que en ningún otro campo. En los ritos agrarios, por ejemplo, se hacen libaciones de chicha ofrendadas a los espíritus de los cultivos y de los animales para asegurar su fertilidad. Estas libaciones van acompañadas de invocaciones verbales: el acto de la ch'alla. Las ch'allas en las comunidades rurales se distinguen por el hecho de que las invocaciones se pronuncian en un registro especial, que se cree apropiado para dirigirse a los interlocutores no humanos. Por lo que pude averiguar, los términos de la ch'alla no son esotéricos, como suele ser el caso del lenguaje ritual en algunas sociedades. Todos los adultos de la comunidad los conocen y pueden informar sobre ellos. En el trabajo de campo solamente pude elicitar los términos; no tuve la ocasión de grabarlos en el momento de su uso ritual. Cabe notar que estos términos y otros similares han sido registrados por varios etnógrafos trabajando en el Norte de Potosi (por ejemplo Arnold, Jiménez y Yapita 1991). El lenguaje ritual, a veces basado en la metáfora, tiene sus raíces mayormente en el aimara y no tanto en el quechua (Cuadro 9). Todos los entrevistados, ya fueran aimarahablantes o no, pensaban que la lengua de las ch'allas "debe ser aimara". Los que hablaban aimara podían ofrecer equivalencias no rituales tanto en aimara como en quechua. En fin, la ch'alla es el campo de acción cultural más arraigado de todos los que venimos estudiando, y es el campo en el cual la lengua de sustrato (ojo con el concepto de sustrato), el aimara escondido del Norte de Potosí, se manifiesta y sobrevive en la práctica más que en ningún otro.

\begin{tabular}{|l|l|l|l|l|}
\hline Aimara & Quechua & $\begin{array}{l}\text { Término } \\
\text { Ritual } \\
\text { phaxsima } \\
\text { mamala }\end{array}$ & $\begin{array}{l}\text { Glosa } \\
\text { luna/pla } \\
\text { ta }\end{array}$ & $\begin{array}{l}\text { Significado Literal } \\
\text { del Término Ritual }\end{array}$ \\
\hline phaxsima & qulqi & papa & 'flaca' \\
\hline ch'uqi & papa & $\begin{array}{l}\text { pana' } \\
\text { t'aqula }\end{array}$ & maíz & 'morena' \\
\hline tunqu & sara & $\begin{array}{l}\text { tirisa } \\
\text { mamala }\end{array}$ & trigo & 'madre Teresa' \\
\hline siwara & tiriju & $\begin{array}{l}\text { wich'inqa } \\
\text { ni }\end{array}$ & cebada & $\begin{array}{l}\text { 'desparramador de } \\
\text { granos' }\end{array}$ \\
\hline alwirija & alwirija & katar layra & alverja & 'ojo de serpiente' \\
\hline apilla & uqa & awki awki & oca & 'anciano' \\
\hline waka & waka & $\begin{array}{l}\text { niñu } \\
\text { tatala }\end{array}$ & vaca & 'padre niño' \\
\hline & & \multicolumn{2}{|l}{} \\
\hline
\end{tabular}




\begin{tabular}{|l|l|l|l|l|}
\hline uwija & uwija & $\begin{array}{l}\text { Luisa } \\
\text { mamala }\end{array}$ & oveja & 'madre Luisa' \\
\hline kawra & kawra & $\begin{array}{l}\text { añawani } \\
\text { mamala }\end{array}$ & cabra & 'madre cascabel' \\
\hline Qarwa & llama & tilantiru & Ilama & 'delantero' \\
\hline
\end{tabular}

Cuadro 9: Términos utilizados en las invocaciones rituales (ch'alla)

\section{Palabras finales}

En el Norte de Potosí las lenguas aimara, quechua y castellano coexisten en un estado de tensión dinámica que nos dice mucho sobre las fuerzas sociales, culturales y de poder que operan en esta región diversa del territorio boliviano. Desde la perspectiva del sector hispano-bilingüe, hay una presión que va en la dirección del modelo urbano, y los préstamos del castellano que se registran en el quechua del pueblo son vistos inclusive como una influencia "modernizante". Al otro extremo del continuum, en la zona rural, el aimara se encuentra sofocado por el quechua. Un examen de los datos comentados aquí sugiere que un modelo de estratificación sociolingüística tripartita (el castellano por encima, seguido por el quechua, seguido por el aimara) no explica todas las tensiones observadas.

Por ejemplo, dentro del mismo quechua hay una jerarquía sociolectal entre el habla urbana y la rural. Esta estratificación, además, se expresa con más claridad en algunos campos léxicos que en otros. En el campo del parentesco, por ejemplo, hay resistencia en contra de la expresión en el quechua urbano de las categorías no hispanas; se remplazan los términos quechuas por hispanismos. En contraste, en el campo de la elaboración de la chicha, el vocabulario quechua no solamente se utiliza en los sociolectos de ambos grupos, sino que también es adoptado para su uso dentro del castellano del pueblo.

El aimara sobrevive en las comunidades alejadas y de acceso difícil desde los caminos y los pueblos, y en contextos de interacción más íntima y esotérica. Tanto mis observaciones de trabajo de campo como los datos lingüísticos recopilados sugieren que el aimara, en esta zona fronteriza entre valle y puna, sirve como código endógeno que refuerza la cohesión social y ayuda a mantener las relaciones armoniosas entre la sociedad humana y los seres del mundo natural animado.

Para regresar al ejemplo con el cual comencé este artículo, llama la atención que la palabra "wirjina", formalmente derivada del castellano pero 
resemantizada en su nuevo contexto, se haya hecho tan prevalente en el campo léxico del lenguaje ritual, que expresa lo más interno de la cosmovisión de los campo runa, campo que, además, fomenta la sobrevivencia del léxico aimara. El uso de "wirjina" para invocar a la tierra animada en las ch'allas, ¿ ¿será un esfuerzo de parte de los campo runa por redibujar la frontera cultural entre su visión del mundo y la de los pueblerinos, una frontera que fue desmantelada de algún modo una vez que los mestizos incorporaron la idea de la pachamama a su cosmovisión? Si vale esta explicación, parece paradójico que los aimaraquechua hablantes del Norte de Potosí recurran a una palabra tan exógena a su sistema de creencias para expresar un concepto tan endógeno. La paradoja es cercana a las que caracterizan el discurso generado en la relación entre el quechua y el castellano desde la época de la colonización más temprana (Pratt 1996). Con la interpenetración entre cosmovisiones y sistemas lingüísticos divergentes, en el acto mismo de hablar, el hablante de las lenguas andinas pierde su autonomía de expresión y, en última instancia, su autonomía de identidad.

\section{Bibliografía Citada}

ADELAAR, Willem. 1986. "La relación quechua-aru: Perspectivas para la separación del léxico". Revista Andina 4(2): 379-426.

ALBÓ, Xavier. 1974. Los mil rostros del quechua. Sociolingüística de Cochabamba. Lima: Instituto de Estudios Peruanos.

ANDRADE CIUDAD, Luis y Rosaleen Howard. (En prensa) "Las lenguas quechuas en los países andino-amazónicos: de las cifras a la acción ciudadana". Revista Arbor.

APPEL, René y Pieter Muysken. 1987. Language Contact and Bilingualism. London, New York: E. Arnold.

ARNOLD, Denise, Domingo Jiménez Aruquipa y Juan de Dios Yapita. 1991. "Scattering the Seeds: Shared Thoughts on Some Songs to the Food Crops". Amerindia 16. 105-178.

BABEL, Anna. 2018. Between the Andes and the Amazon: Language and Social Meaning in Bolivia. Tucson, AZ: University of Arizona Press.

CERRÓN-PALOMINO, Rodolfo. 2008. Quechumara. Estructuras paralelas del quechua y del aimara. La Paz: UMSS/PROEIB Andes/Plural Editores.

COLLINS, JANE. 1983. "Translation Traditions and the Organization of Productive Activity: The Case of Aymara Affinal Kinship Terms". Bilingualism: Social Issues and Policy Implications. Andrew W. Miracle, ed. Athens, GA: Georgia University Press. 11-22.

HARRIS, Olivia. 1985. "Ecological Duality and the Role of the Center: Northern Potosí". Andean Ecology and Civilization: An Interdisciplinary 
Perspective on Andean Ecological Complementarity. Yoshio Mazuda, Izumi Shimada y Craig Morris, eds. Tokyo: University of Tokyo Press. 311-335.

---. y Xavier Albó. 1986. Monteras y guardatojos. Campesinos y mineros en el Norte de Potosí en 1974. Cuadernos de Investigación (Centro de Investigación y Promoción del Campesinado) 26. La Paz: CIPCA.

HILL, Jane H. y David M. Coombs. 1982. "The Vernacular Remodelling of National and International Languages". Applied Linguistics III(3): 224234.

HOWARD, Rosaleen. 2007. Por los linderos de la lengua. Ideologías lingüísticas en los Andes. Lima: Instituto de Estudios Peruanos/Instituto Francés de Estudios Andinos/Editorial de la PUCP.

---. $\quad$ et al. 2014. Kawsay Vida: A Multimedia Quechua Course for Beginners and Beyond. Austin, TX: University of Texas Press.

HOWARD-MALVERDE, Rosaleen. 1995. "Pachamama is a Spanish Word": Linguistic Tension between Aymara, Quechua, and Spanish in Northern Potosí (Bolivia)". Anthropological Linguistics 37(2): 141-168.

LASTRA, Yolanda. 1968. Cochabamba Quechua Syntax. The Hague, Paris: Mouton.

MOLINA, Ramiro y Xavier Albó. 2006. Gama étnica y lingüística de la población boliviana. La Paz: Programa de las Naciones Unidas para el Desarrollo.

PRATT, Mary Louise. 1996. Apocalypse in the Andes. Contact Zones and the Struggle for Interpretive Power. Lecture. Washington, DC: InterAmerican Development Bank (IDB) Cultural Center. Encuentros 15. March 1996.

REPÚBLICA DE BOLIVIA. 1993. Censo Nacional de Población y Vivienda 1992. Resultados finales. La Paz: Ministerio de Planeamiento y Coordinación, Instituto Nacional de Estadística.

SICHRA, Inge. 2003. La vitalidad del quechua: Lengua y sociedad en dos provincias de Cochabamba. La Paz: PROEIB Andes/Plural Editores.

YAPITA, Juan de Dios, Denise Arnold y Elvira Espejo. 2014. Los términos textiles aymaras actuales de la región Asanaque: Vocabulario semántico según la cadena productiva. La Paz: Instituto de Lengua y Cultura Aymara/Fundación Xavier Albó.

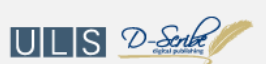

New articles in this journal are licensed under a Creative Commons Attribution 4.0 United States License.

This journal is published by the University Library System of the University of Pittsburgh as part of its D-Scribe Digital Publishing Program, and is cosponsored by the University of Pittsburgh Press. 